\title{
Development and application of diagnostic tools for seawater intrusion analysis in highly heterogeneous coastal aquifers
}

\author{
M. Adil Sbaï ${ }^{1}$ and N. Amraoui ${ }^{1}$ \\ ${ }^{1}$ Water Environment and Ecotechnologies Division, BRGM, Orléans, France
}

\begin{abstract}
We developed novel, computationally efficient, methods as effective screening tools to analyze seawater intrusion processes in highly heterogeneous coastal aquifer systems. They enable delineation of pumping wells capture zones and swept zones associated to injection wells for remediation of seawater encroachment. Forward or backward travel times and residence time distributions are robustly simulated and visualized on the computational grid. These steady-state indicators, precomputed at fine grids, are used to generate optimal locally refined grids for efficient transient solute transport simulations.
\end{abstract}

\section{INTRODUCTION}

A large array of sweater intrusion (SI) processes may develop in hydrogeological environments. These effects include among others dispersive mixing, density and viscosity effects, unstable convective mixing, tidal effects, aquifer recharge variability, surfacesubsurface interactions, and geological controls. The interplay between all these processes is a challenge for current generation of groundwater flow and transport models. One of the most challenging and still largely unresolved aspects of SI prediction is representation of the local geological features controlling SI. Only a handful set of well-documented case studies tackled the problem to highlight the influence of aquifer heterogeneity on SI processes.

Therefore, new methods have to be developed yet to address the shortcoming of traditional modeling approaches. Here we present a suite of diagnostic tools to fill existing gaps when analyzing SI processes in highly heterogeneous coastal aquifers. These methods are computationally cheap and equipped with novel visualization capabilities to enhance interpretation and understandings of flow and transport processes. They could be easily used at different stages of building, calibration, or updating seawater intrusion models. Two examples are given to illustrate their advantages when used in conjunction with a sharp interface or coupled flow and transport approaches.

\begin{abstract}
APPROACH
The computational approach is an extension of a newly proposed grid-based alternative method to advective particle tracking (Sbai 2018). This is a powerful screening tool to accurately delineate and visualize capture zones around abstraction wells or outflow boundaries, the swept zones formed by injection wells or inflow boundaries, and the partitions associated with injection-pumping well doublets or inflowing-outflowing boundary pairs. The forward or backward travel times and residence time distributions are robustly simulated and visualized on the computational grid with little computational effort. This method is comparable to the particle-tracking approach (Pollock 1988). However, it holds more promise for complex groundwater applications because the interpretation and visualization of travel and residence times are easier.
\end{abstract}


The first extension proposed in this paper involves the interactions between the sea boundary and all sources/sinks of a sharp-interface groundwater flow model. A second addition is a novel way to generate optimal locally refined grids (LGR) for transient solute transport problems based on precomputed steady-state indicators derived from a fine scale steady-state flow and travel times solutions.

\section{RESULTS}

Two examples are presented to demonstrate the usefulness of the proposed extensions for seawater intrusion analysis in coastal aquifers.

\section{Example 1}

This first demonstration example considers four pumping wells in a highly heterogeneous hypothetical coastal aquifer system. As illustrated in Figure 1A the permeability distribution spans more than five orders of magnitude. An injection well is placed in the south of the domain near the coastline at the lower boundary. The effectiveness of this hydraulic barrier, over time, could be studied by computing and visualizing the expected spatial-temporal patterns of the flow field. A more effective way to understand the dynamics of hydraulic interactions between the injection well, pumping wells, and the salt-fresh water interface is to analyze the shape of swept zones corresponding to forward tracer partitions from the injector.

(A)

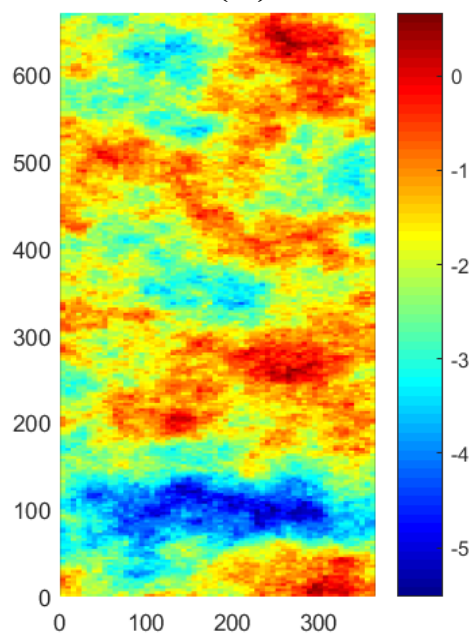

(B)

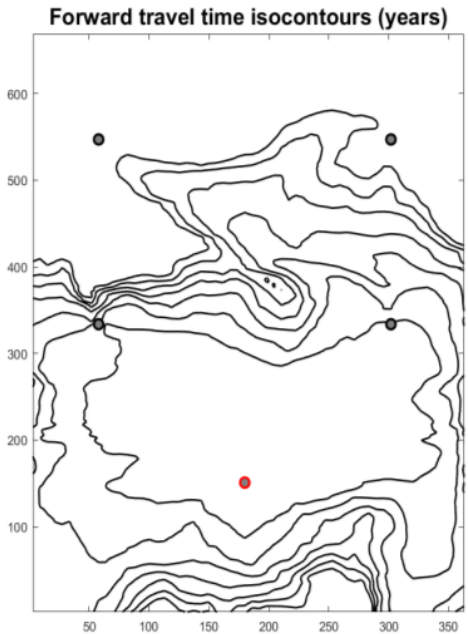

(C)
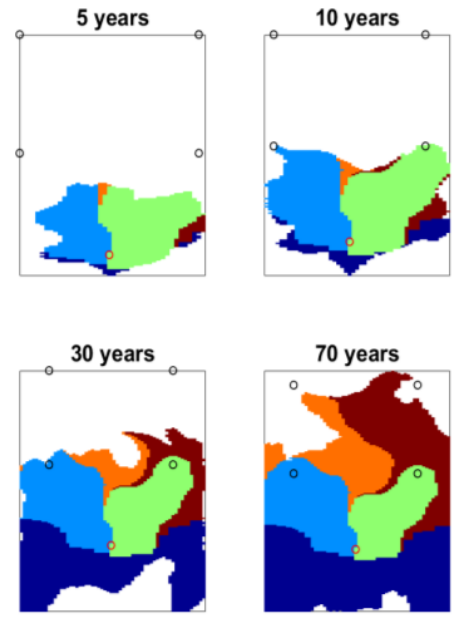

Figure 1. Permeability distribution in $\log _{10}$ scale. (B) Forward time iso-values from 10 to 70 years with 10 years interval. Injection well is red and pumping wells are black dots. (C) Spatial-temporal patterns of swept zones formed by the injection well showing the strength and magnitude of hydraulic interactions between injection-pumping well pairs and its potential to remediate saltwater encroachment.

Figure 1B shows the contour lines of the forward arrival times simulated by a finitedifference grid-based method (Sbai 2018). Injected water reaches the pumping wells in the domain center and north positions after 10 and 70 years, respectively. Figure $1 \mathrm{C}$ shows the expansion of interaction zones between the injector and other sources/sinks such as the salt source from the sea and the pumping stations. In particular, we can conclude that with the prescribed flow rates remediation of seawater intrusion is starting to be effective after 30 years (notice the expansion of the dark blue partition in Figure 1C). 
Because these results are obtained very efficiently (in less than a second in a laptop computer) the underlying method could be used for operational management of water resources in coastal aquifers. It could also be used in combination with a constrained optimization algorithm to design the optimal number, positions and flow rates of injection wells.

\section{Example 2}

In this second demonstration example we consider a binary distribution of the hydraulic conductivity field as shown in Figure 2A where permeable and impervious cells are at 1 and $10^{-3} \mathrm{~m} / \mathrm{d}$, respectively. Groundwater flow is controlled by a couple of injection and pumping wells placed at the lower left and upper right cells of the computational grid, respectively. Pumping and withdrawal rates are equal to $1 \mathrm{~m}^{3} /$ day. Effective porosity equals $25 \%$.

Groundwater flow and finite-difference based forward and backward travel times are simulated on a fine grid with $1 \mathrm{~m}$ uniform grid spacing in each direction. Next, a fine scale spatial distribution of $I_{\tau}=-\left[\log \left(\tau_{f}\right)+\log \left(\tau_{b}\right)\right]$ is computed and shifted to the origin as illustrated in Figure 2B.

A general-purpose algorithm is developed to optimize the generation of LGR grids for transient solute transport simulations in heterogeneous porous media. The novelty of this method is its reliability on physical (flow and transport) dynamics for grid generation unlike generally available tools standing rather on geometrical constraints. This algorithm involves two distinct steps. First, the fine scale grid is coarsened by a regular agglomeration of the cells following an $n \times m$ pattern, where $n$ and $m$ are the number of successive rows and columns to agglomerate. This step involves eventually a flow-based upscaling procedure to get representative hydraulic conductivity values for the obtained coarse grid. In a second phase, coarse blocks whose cumulative $I_{\tau}$ index exceed a given threshold value $\tau_{c}$ are taken as local grid refinement targets. A user-selected grid refinement level is therefore applied to all these cells leading to a fine child grid having inactive cells which overlap with active cells of its parent coarse grid, and vice-versa. Flow and transport simulations involve an iterative loop between the parent and child grid(s) where jumps in flow rates and concentration fluxes at their interfaces are minimized until convergence. This is similar to a domain decomposition method at the algebraic level. An example of such generated grid is shown is Figure 2C for the presented problem where $n=m=5$ and the refinement level inside the child grid is 5-by-5.

As shown in Figures 3A and 3B groundwater heads computed with the fine and LGR grids look very similar in sensitive parts to solute transport dynamics namely inside the fine child grid of the composite LGR grid. As expected pure solute advection simulations results computed on fine and LGR grids are undisguisable as illustrated in Figures 3C and 3D resulting in overall reduction of the computational time and memory storage. This conclusion is confirmed by comparing the breakthrough curves of the solute at the pumping well shown in Figure 3E where an excellent agreement is obtained.

We expect a more impressive speedup for three dimensional problems in heterogeneous aquifers exhibiting high flow channeling or localized solute transport spreading. This is also the case for seawater intrusion problems based on coupled viscosity- and density-dependent flow and transport models because repeatedly solving the flow problem on an LGR grid could dramatically reduce the computational time and accelerate the outer (e.g. nonlinear) 
loop convergence. All these issues are being under investigation and will be reported in the near future.

(A)

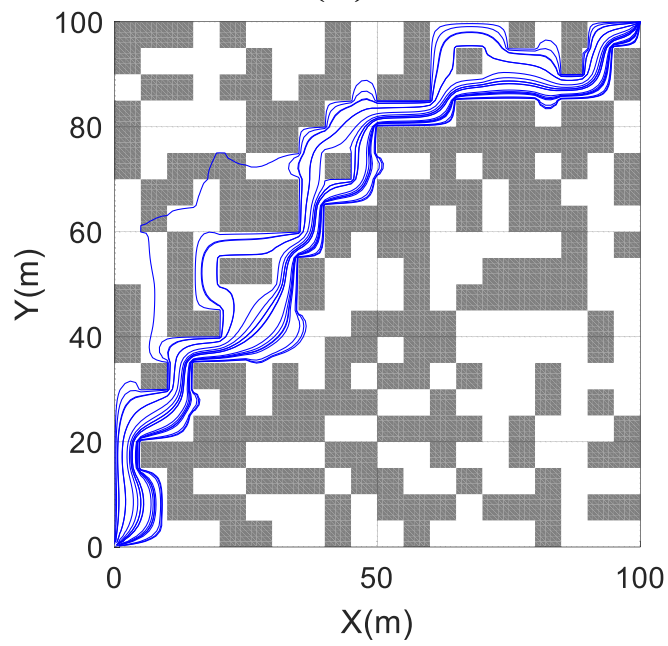

(B)

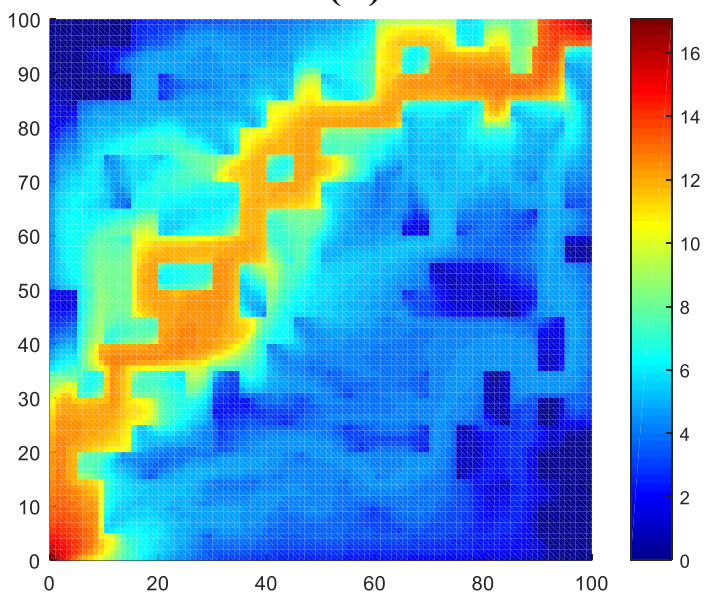

(C)

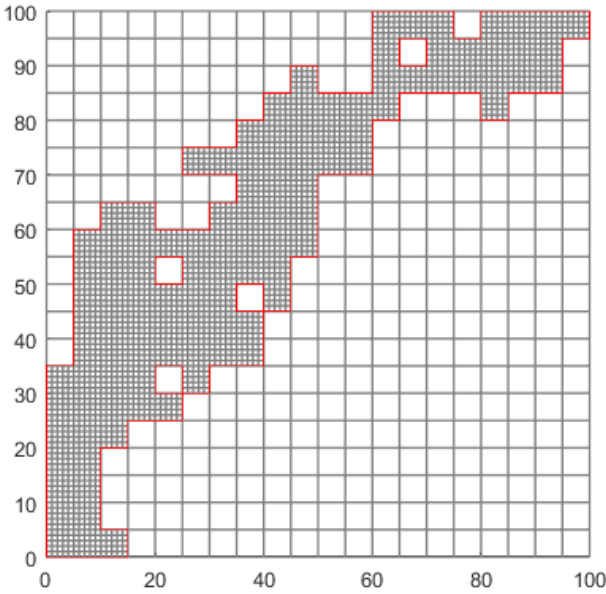

Figure 2. Hydraulic conductivity distribution showing impervious cells in gray. Flow pathlines of 20 particles released near the injection well are visualized. (B) Spatial distribution of $I_{\tau}$ indicator used for local grid refinement. (C) The obtained LGR grid to be used for the following transient solute transport simulation with $\tau_{c}=\mathbf{3 0}$.

\section{CONCLUSIONS}

We introduce novel screening tools to analyze seawater intrusion processes in highly heterogeneous coastal aquifer systems. Illustrative examples were given to demonstrate:

- The capability to delineate the spatial-temporal interactions between the sea boundary and all sources/sinks of a sharp-interface groundwater flow model.

- The capability to generate optimal locally refined grids for transient solute transport problems based on precomputed steady-state indicators. Thus, we introduce a groundwater flow- and transport- based method for grid generation targeting finite difference models.

We are using these diagnostic tools to analyze and improve conceptual models of existing Mediterranean seawater intrusion pilot sites in South of France. 
(A)

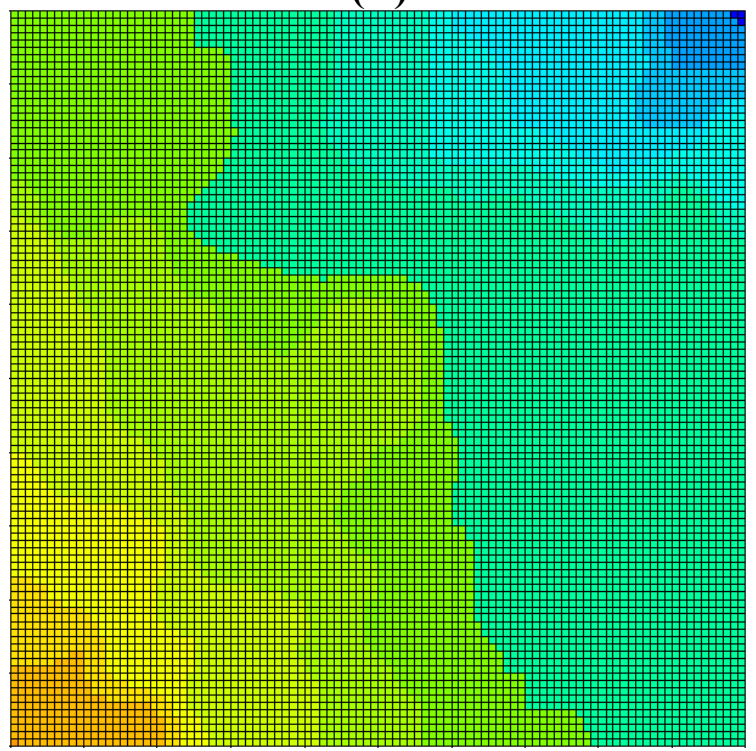

(C)

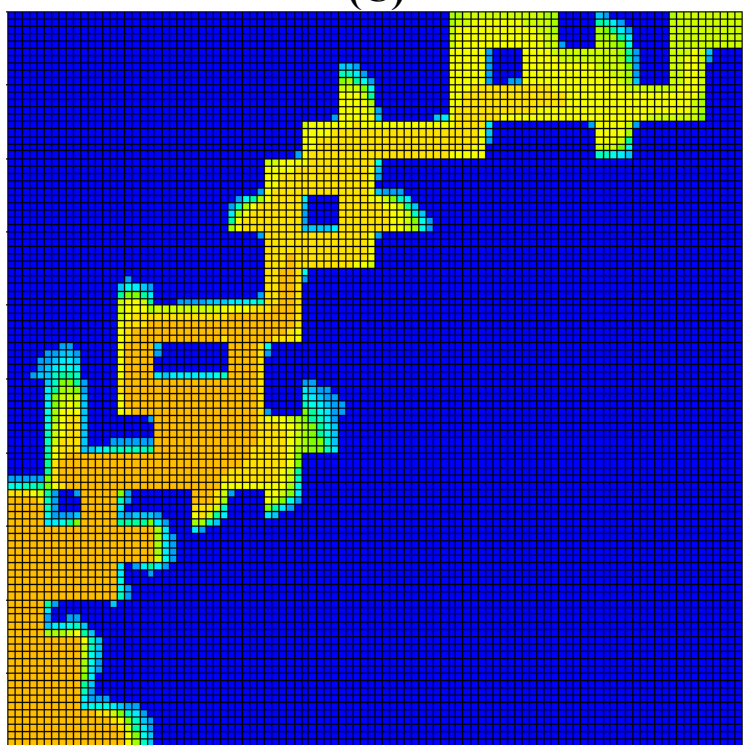

(E)

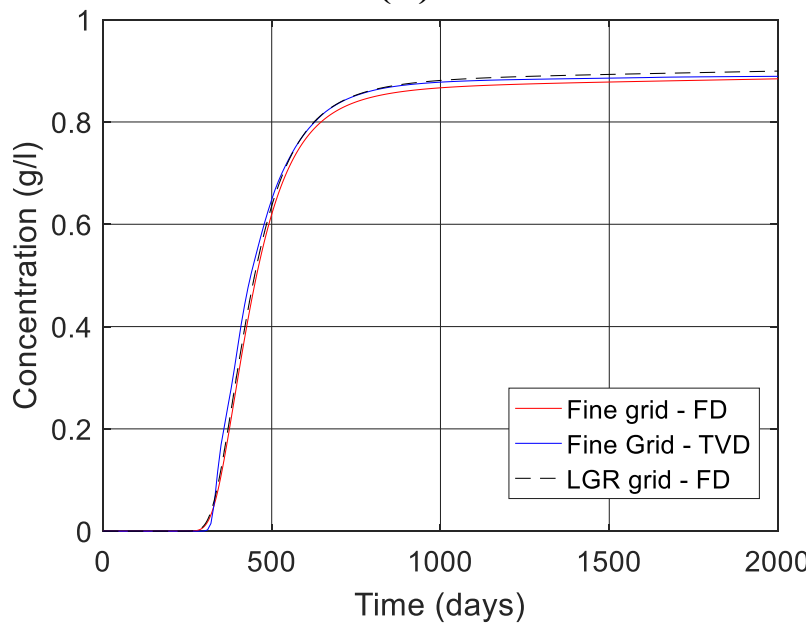

(B)

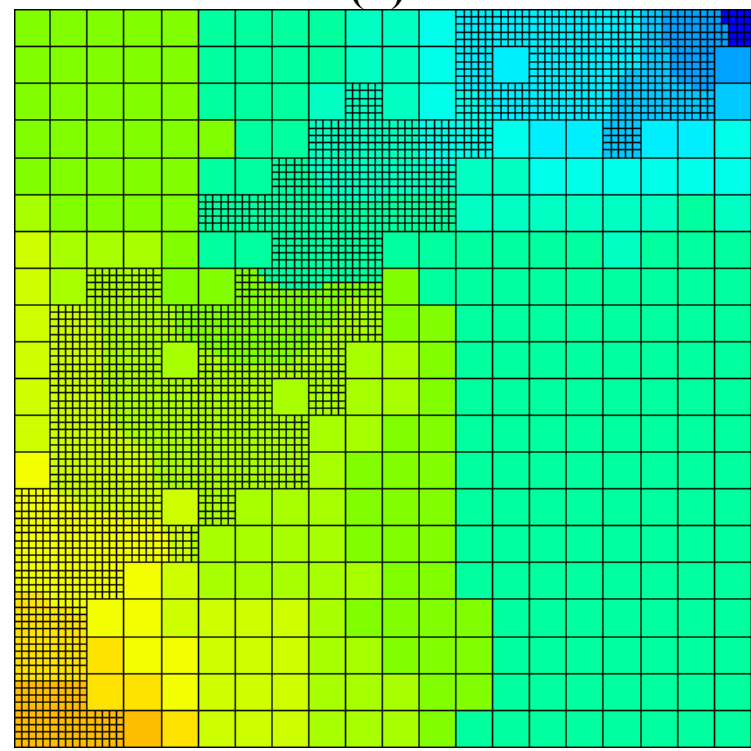

(D)

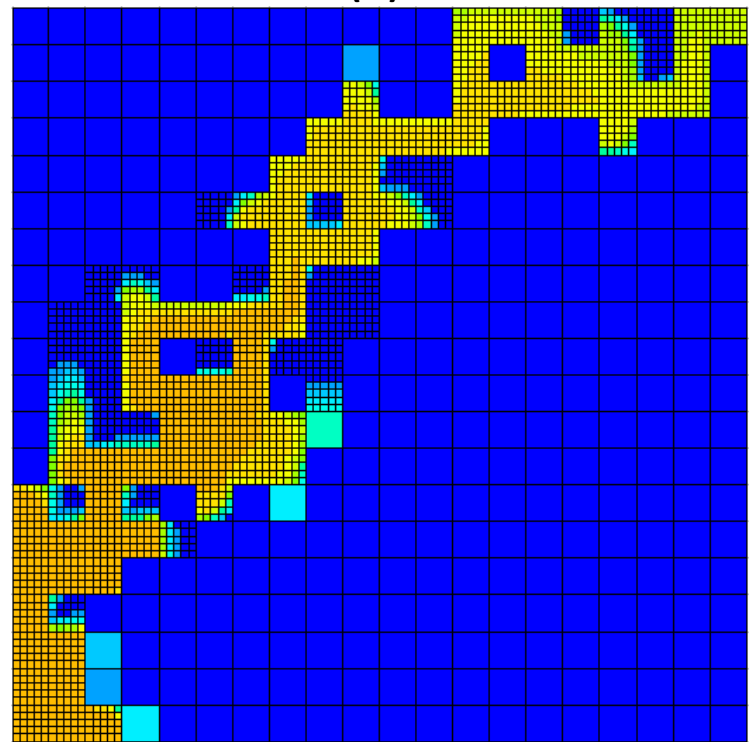

Figure 3. Spatial distributions of computed hydraulic heads on (A) fine and (B) LGR grids. Spatial distribution of simulated concentrations after 600 days of continuous injection/pumping using (C) fine and (D) LGR grids. A

CPU time speedup close to two is obtained. (E) BTC's at the pumping well are shown for fine and LGR grids, respectively. Two fine grid solutions are produced with Finite-Difference (FD)

and TVD schemes. An excellent agreement is obtained between the three simulations. 


\section{REFERENCES}

Pollock, D.W. 1988. Semianalytical computation of path lines for finite difference models, Groundwater 26(6), 743-50.

Sbai, M.A. 2018. A practical grid-based alternative method to advective particle tracking. Groundwater, accepted.

Contact Information: M. Adil Sbaï, BRGM (French Geological Survey), Water Environment and Ecotechnologies Division, Water Management Group, 3 Avenue Claude-Guillemin, BP 36009, 45060 Orléans Cedex 2, France, Phone: +33.2.38.64.35.27, Fax: +33.2.38.64.37.19, Email:

a.sbai@brgm.fr 